\title{
The Safety and Efficacy of the Indwelling Valve Catheters in the Long-term Catheterised Patients: A Systematic Comparative Study
}

\author{
Omer A. Raheem Rowan G. Casey Frank T. D'Arcy Thomas H. Lynch \\ Department of Urology, St James's Hospital, Dublin, Ireland
}

\section{Key Words}

Valve catheter • Non-valve catheter • Safety • Efficacy

\begin{abstract}
Introduction: There is paucity of data regarding the safety and efficacy of the valve catheters (VC). We aimed to prospectively evaluate our experience in regards to the safety and efficacy of the VC and compared it to patients with nonvalve catheters (NVC). Methods: Total 76 male patients were included in both the VC and NVC groups. The type of catheters was standardized by using Bard ${ }^{\circledR}$ manufacturer, United Kingdom. Patients were evaluated 4-6 weeks following catheterization by filling out a non-validated quality of life (QoL) questionnaire at their scheduled clinic visit. Results: Mean age was 75 years (range 62-85 years) and 77 years (range 66-88 years) in the VC and NVC groups respectively. There were comparable results of the VC and NVC groups. However, observed benefits were found in regards to the ease (4/5-point scale: $64 \%$ in VC and $53 \%$ in NVC), comfort of usage (4/5-point scale: $59 \%$ in VC and $54 \%$ in NVC), patient's confidence (very confident: $95 \%$ in VC and $82 \%$ in NVC) ( $p>$ 0.05). Three bladder spasms were recorded in the VC group, compared to 2 in the NVC group. In contrast, only 1 urinary tract infection observed in the VC group, compare to 2 in the NVC group ( $p>0.05)$. Conclusions: This study demonstrates that VC is a viable and patient friendly alternative. This may be partly explained by the functional mechanism of the VC mimicking the urinary bladder sphincters. Future research should aim to further improve its mechanical functionality.
\end{abstract}

Copyright ๑ 2011 S. Karger AG, Basel

\section{KARGER}

Fax +4161306 1234

E-Mail karger@karger.ch

www.karger.com
(C) 2011 S. Karger AG, Basel

1015-9770/11/0054-0173\$26.00/0

Accessible online at:

www.karger.com/cur

\section{Introduction}

Bladder drainage via indwelling catheters is usually indicated in patients with short-term bladder outlet obstruction such as benign prostatic hyperplasia (BPH). However, failing the utilization of intermittent self-catheterization, a long-term form of bladder drainage is often directed to elderly patients with neurogenic bladders [1, 2]. Neurogenic bladder can result from a wide range of causes such as inflammatory, ischemic and traumatic i.e. transverse myelitis, cerebro-vascular accidents and spinal injuries $[1,2]$. Continuous bladder drainage via an indwelling catheter attached into a leg bag i.e. non-valve catheters (NVC) remains an effective method of emptying the bladder for many patients. More recently, there has been increasing evidence in the published literature and clinical practice to support using an alternative method of bladder drainage such as valve catheters (VC). A $\mathrm{VC}$ is a small drainage tap that fits into the end of the indwelling catheter. Hence, urine is stored in the bladder rather than in a bag attached to the leg which maintains bladder muscle tone and pressure and allowing cycling of the bladder by releasing the valve on regular intervals [1-3].

The introduction of $\mathrm{VC}$ has given catheterized patients choice and widened the range of therapeutic interventions that are available, particularly, reduced urinary tract infection (UTI), maintenance of bladder tone, function and capacity as well as patient's preference [4-6]. Previous studies have described the 'flushing effect' which may reduce infection when the bladder is emptied periodi- 


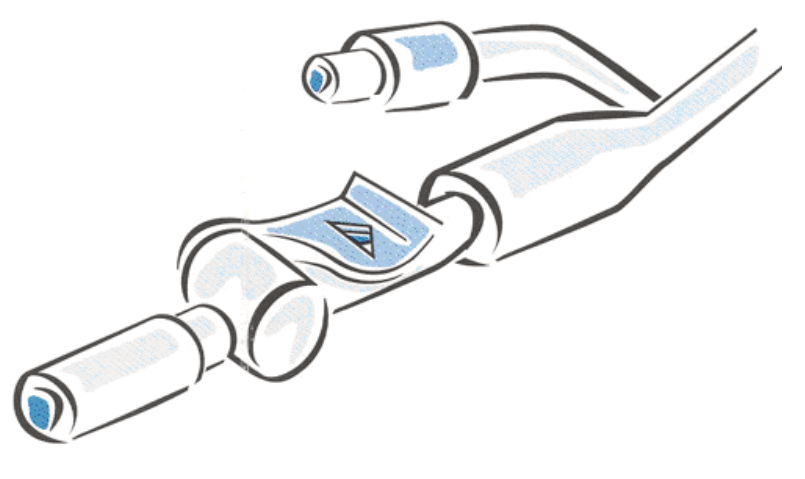

Fig. 1. Schematic drawing of the valve catheter (Flip-Flo ${ }^{\circledR}$, Bard Ltd, UK) demonstrating the mechanical valve attachment with the indwelling urinary catheter (urinary bag not attached).

Table 1. Patients' demographics and details in the VC and NVC groups

\begin{tabular}{lll}
\hline & VC & NVC \\
\hline Patients, n & $38(100 \%)$ & $38(100 \%)$ \\
Mean age (range), years & $75(62-85)$ & $77(65-88)$ \\
Sex & Male (100\%) & Male (100\%) \\
Catheter manufacturer & Bard, UK & Bard, UK \\
Follow-up period, weeks & $4-6$ & $4-6$ \\
\hline
\end{tabular}

cally. Where infection exists, catheter blockage is probable therefore the same 'flushing effect' may, in theory, reduce blockages [4-6]. Regarding comfort, numerous studies agree that the VC is superior to the NVC. Notably, NVC has the potential to create heat rash although some bags have a fabric backing or sleeve to prevent this effect to some limited extent. Furthermore, vast majority of the VC including Flip-Flo ${ }^{\circledR}$ (Bard, United Kingdom), have the potential to form a link system, allowing the patient to easily attach urinary collecting bag to drain urine out [4-6].

We sough to prospectively evaluate the safety and efficacy of the VC in elderly men with long-term catheters and compare it prospectively to patients with NVC.

\section{Patients and Methods}

Over a year period, a total of 76 male patients were included in both the VC and NVC groups (38 male patients in each group).
All patients required acceptable dominant hand function to manage their respective urinary catheters. The hand functions were clinically assessed by the table top test in each patient. The type of VC and NVC was standardized by using Bard $^{\circledR}$ manufacturer, United Kingdom (UK) (fig. 1). No patients in both groups developed UTIs in the year period preceded the utilization of the urinary catheters. Patients were evaluated 4 to 6 weeks following catheterization by filling out a non-validated quality of life (QoL) questionnaire given by urology specialist nurse at their scheduled clinic visit (appendix 1). The QoL questionnaire was designed to record VC and NVC performances by patients on their scheduled review dates. Patients' demographics and details are summarized in table 1 .

QoL questionnaire consisted of the level of satisfaction, easy of use, hand function, valve open and closing function, comfort of use, inconspicuous, patient confidence using catheter valve at social places were all scored against 5-point scale of excellent, very good, good, fair and poor. The frequency and treatment of bladder spasm and/or by-passing/blockages of the catheters were also assessed. The frequency of changing the catheters in both groups was also observed as well as the utilization of nocturnal urinary drainage bags. When clinically indicated, urinalysis and/ or urine cultures were also performed by the urology nurse. The presence of UTIs from a catheter specimen of urine and subsequent antimicrobial treatment were also recorded. Comparative analysis between the 2 groups was performed using fisher's exact. $\mathrm{P}$ value $<0.05$ considered to be statistically significant. All data were collected and stored electronically. Comparative results are summarized in table 2.

\section{Results}

Mean age of the VC group was 75 years (range $62-85$ years), whereas mean age of the NVC group was 77 years (range 66-88 years). The majority of patient's level of satisfaction was recorded as 4/5-point scale in $53 \%$ of the VC group an $51 \%$ of the NVC group ( $p>0.05)$. Higher number of patients found the usage of the VC easier (4/5-point scale), compared to the usage of NVC (64 and $53 \%$ respectively) $(\mathrm{p}>0.05)$. Dominant hand function was observed as good function in all patients (3/5-point scale) and valve open and closing mechanism of the VC was also assessed and found to be very good (4/5-point scale) in $53 \%$ of patients. Comfort of usage was found to be very good (4/5-point scale) in 59\% of VC group and $54 \%$ of NVC group ( $p>0.05)$. Similarly, inconspicuous was also higher in the VC group (very good, 4/5-point scale) at $63 \%$, compared to the NVC group (very good, $4 / 5$-point scale) at $53 \%(\mathrm{p}>0.05)$. Ninety-five percent of patients were very confident of utilizing the VC, compared to $82 \%$ in the NVC group ( $p>0.05$ ).

Bladder spasms was recorded in 3 patients $(8 \%)$, ranged between 2 and 6 spasms per day in the VC group, compared to 2 patients (5\%), ranged between 1 and 4 spasms 
Table 1. Comparative results of the VC and NVC groups

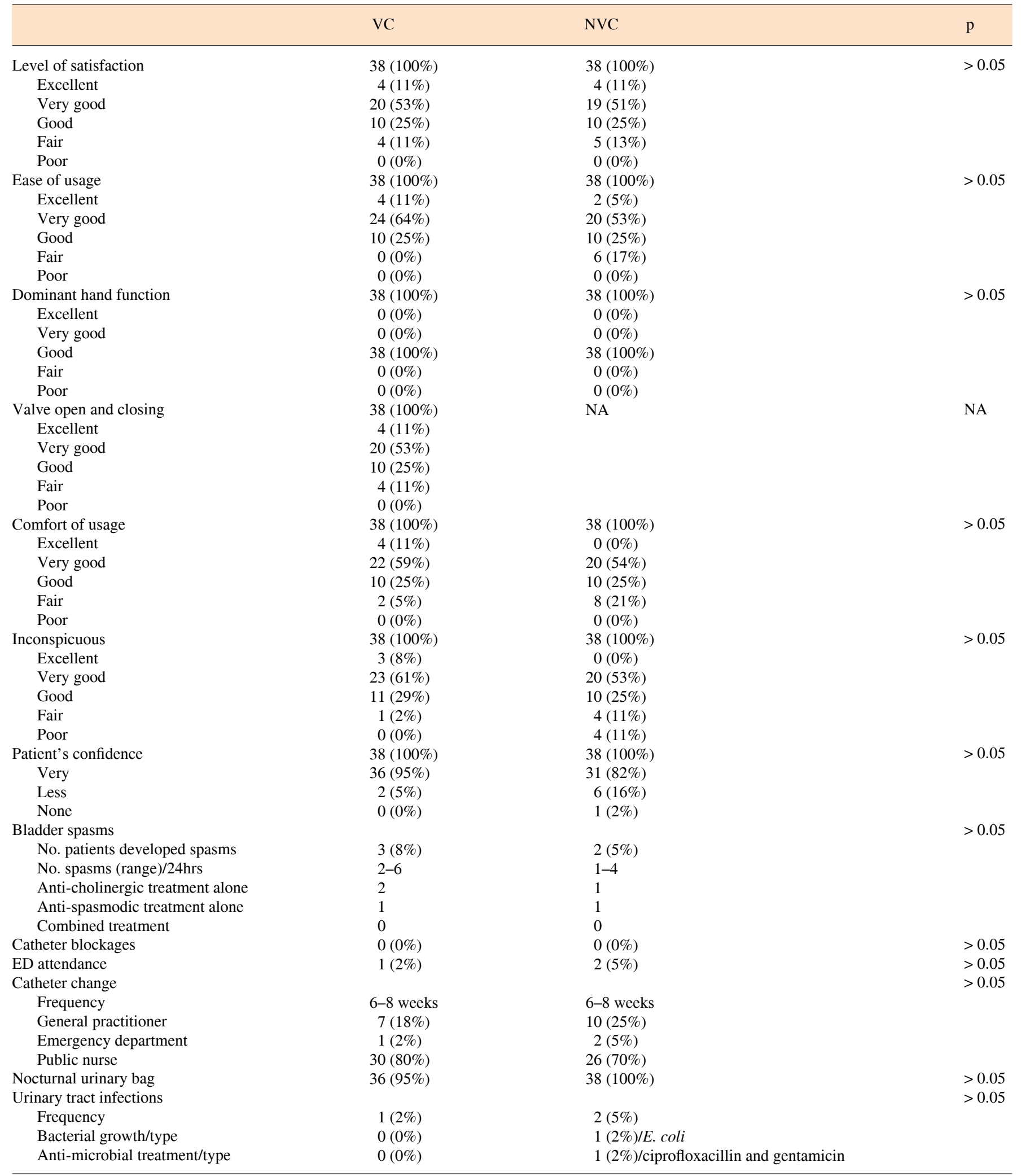

$\mathrm{NA}=$ Not available 
per day in the NVC group. Three patients required treatment for bladder spasms in the VC group, compared to 2 patients in the NVC group. Anti-cholinergic medications was prescribed in 2 patients in the VC group, whereas, only 1 patient was on anti-cholinergic in the NVC group. Anti-spasmodic was prescribed in 1 patient only in each group. No patient required combined therapy. Time of catheter change was usually after 6-8 weeks and commonly preformed by the public nurse $(80 \%$ in the $\mathrm{VC}$ group and $70 \%$ in the NVC group) ( $\mathrm{p}>0.05)$.

Ninety-five percent of patients utilized noctural urinary bag to manage their $\mathrm{VC}$, hence avoiding the trip to bathroom during the nighttime. One patient developed UTI with no identified bacterial growth in the urine in the VC group, compared to 2 patients in the NVC group $(\mathrm{p}>$ 0.05 ). Among the 2 patients developed UTIs in the NVC group, 1 patient had $E$. coli UTI requiring anti-microbial medications of ciprofloxacillin and gentamicin.

\section{Discussion}

Although, there is a paucity of research-based literature on VC, an increasing body of evidence suggests that many patients would prefer a VC over a NVC, in particular to level of satisfaction and comfort [6-13]. Adding to this, numerous comparative studies have aimed to compare the use of $\mathrm{VC}$ to $\mathrm{NVC}$ and conclusions have been drawn that the $\mathrm{VC}$ was extremely acceptable to the catheterized patients with no significant difference in the prevalence of catheter-related complications, particularly the development of UTIs. It is also believed that the 'flushing effect' of VC may reduce the development of UTIs when the bladder is emptied periodically and frequently. Wilson et al. [12] compared different types of catheters and found less prevalence of cross-infection among VC groups.

German et al. [9] conducted a randomized, crossover trial of VC versus NVC on 16 male patients over 3-week period. They found that the $\mathrm{VC}$ was more comfortable than the NVC during walking and enabled more natural voiding mechanism. However, in the VC group, there was more nocturnal frequency and episodes of urge incontinence observed. Additionally, they concluded that the ideal management would be a VC during the day and a nocturnal urinary bag for drainage. It was also reported that the NVC had the potential to create heat rash on the thigh or upper leg area.

Fader et al. [3] have shown that the CV can be both utilized urethrally or suprapubically. The VC can also al- low men to use a urinal standing up to empty their bladders [9]. In a study by Bard manufacturer, patients who regularly utilized the $\mathrm{VC}$ achieved better voiding restoration following their prostate surgery i.e. transurethral resection of prostate [1]. On removal of the $\mathrm{VC}$, the restoration of normal or near normal voiding functions can be partly explained by the maintenance of the bladder tone generated by the VC mechanism $[11,13]$.

Generally, the use of catheters may cause trauma to the bladder as well as urethra. Roe et al. [8] described erosions of the bladder mucosa caused by catheter's irritation and actual sores can develop in the bladder, caused by the friction of a catheter. The $\mathrm{VC}$ may reduce this risk by periodically lifting the bladder wall away from the catheter and allowing the bladder to refill. Additionally, bladder neck traction may also be minimized by the VC [2].

Previous reported studies have shown that bladder shrinkage can manifest after a period of 6 months or over if on continuous urinary drainage ensured by NVC [4]. In patients utilizing $\mathrm{VC}$, bladder function, capacity and tone can be maintained [1]. However, the long-term usage of the $\mathrm{VC}$ can eventually lead to development of bladder spasms (detrusor instability), by-passing of catheter and catheter blockages $[8,9]$. It is also worth mentioning that VC should be avoided in patients with known vesicoureteric reflux and chronic renal impairment $[3,6]$.

In the present study, there have been comparable results of the VC and NVC groups (table 2). However, potential benefits were observed in regards to the ease, comfort of usage, patient's confidence in the VC group compared to the NVC group. Although, more bladder spasms occurred in the VC group, all patients responded very well to the medical treatment. In contrast, there were more UTIs developed in the NVC, in comparison to the VC group.

The observed benefits of the clinical utilization of the VC can be partly due to their functional mechanism which mimics the normal physiological control of the urinary bladder sphincters. Moreover, the dexterity functions of the patients using $\mathrm{VC}$ need to be assessed in order to achieve satisfactory results.

\section{Conclusion}

The VC is a viable and patient-friendly alternative to the NVC in patients with long-term catheters. Future research studies should be directed to further improve the function of VC, perhaps with utilization of electrical sen- 
sors allowing synchronized and timed movement of the valve in the catheter to the bladder pressure. Adding to this, patients' functional and voiding outcomes follow- ing trail without $\mathrm{VC}$ and/or surgery should also be considered as end-points in the future studies.

\section{Appendix 1 \\ Non-validated Quality of Life (QoL) questionnaire utilized to asses safety and efficacy of the valve and non- valve catheters \\ Department of Urology \\ Please attach Patient sticker here \\ St' James's Hospital, Dublin, Ireland}

Quality of Life (QoL) questionnaire for the valve and non-valve catheters study

Date: --/--/----

Please circle as appropriate

1. Type of catheter valve? $\quad$ Valve catheter Non-valve catheter

2. Are you satisfied with the valve or non-valve catheter? Excellent Very good Good Fair Poor

3. Do you find it Easy to use? $\quad$ Excellent $\quad$ Very good Good Fair Poor

4. How is your hand function? $\quad$ Excellent $\quad$ Very good Good Fair Poor

5. Do you find it easy to open and close? Excellent $\quad$ Very good Good $\quad$ Fair Poor

6. Is the valve catheter comfortable to wear? Excellent $\quad$ Very good Good Fair Poor

7. Is it inconspicuous (not noticeable)? $\quad$ Excellent $\quad$ Very good $\quad$ Good $\quad$ Fair $\quad$ Poor

8. Are you confident using the valve catheter outside home and at social places? Very Less None

9. Have you experienced bladder spasms? Yes $\quad$ No

10. How many bladder spasms during 24 hours? $11 \quad 2 \quad 3 \quad 3 \quad 4 \quad 5 \quad>5$

8. What treatment have you had for bladder spams? Anti-cholinergic Anti-spasmodic Combined

9. Have you experienced catheter blockages? Yes Now many in 24 hours? Number?

10. Have you attended Emergency department for catheter related problems? Yes No

11. How often you change catheter? Where? Change General practitioner Emergency department Public nurse

12. Do you use urinary bag during night? $\quad$ Yes No

13. Have you had urinary tract infections (UTI)? Yes No

14. Have you been on antibiotics for UTI? Yes No

Please return this questionnaire to the urology nurse - Thank you for participation in this study 


\section{References}

1 Bard: Flip-Flo: providing patients with greater independence and improved surgical outcome. Urol News 1998;3:33.

$>2$ Doherty W: The Sims Portex Catheter Valve: an alternative to the leg bag. Br J Nurs 1999; 8:459-462.

3 Fader M, Pettersson L, Brooks R, Dean G, Wells M, Cottenden A, Malone-Lee J: A multicentre comparative evaluation of catheter valves. Br J Nurs 1997;6:359-367.

4 Kristiansen P, Pompeius R, Wadstrom LB: Long-term urethral catheter drainage and bladder capacity. Neurourol Urodyn 1983;2: 135-143.
5 Oberst MT, Graham D, Geller NI Stearns MW Jr, Tiernan E: Catheter management programs and postoperative dysfunction. Res Nurs Health 1981;4:175-181.

6 Pettersson L, Fader M: Choosing a catheter valve that suits the patient. Community Nurse 1997;3:9.

7 Roe B: Do we need to clamp catheters? Nurs Times 1990;86:66-67.

8 Roe B: Catheterization; in Norton C (ed): Nursing for continence, 2 ed. Beaconsfield Publishers, Beaconsfield, 1996, pp207.

$\checkmark 9$ German K, Rowley P, Stone D, Kumar U, Blackford $\mathrm{HN}$ : A randomized cross-over study comparing the use of a catheter valve and a leg-bag in urethrally catheterized male patients. Br J Urol 1997;79:96-98.
10 Wilson C, Sandhu SS, Kaisary AV: A prospective randomized study comparing a catheter valve with a standard drainage system. Br J Urol 1997;80:915-917.

11 Williamson ML: Reducing post-catheterization bladder dysfunction by reconditioning. Nurs Res 1982;31:28-30.

12 Wilson M, Coates D: Infection control and urine drainage bag design. Prof Nurse 1996; 11:251-252.

13 Addison R: Catheter valves: a special focus on the Bard Flip-Flo catheter. Br J Nurs 1999;8:576-580. 